\title{
Diagnostic Criteria for Mild Cognitive Impairment in Parkinson's Disease: Movement Disorder Society Task Force Guidelines
}

\author{
Irene Litvan, $\mathrm{MD}^{1}{ }^{1,}$, Jennifer G. Goldman, MD, $\mathrm{MS}^{2}$, Alexander I. Tröster, $\mathrm{PhD}^{3}$, Ben A. \\ Schmand, $\mathbf{P h D}^{4}$, Daniel Weintraub, MD $^{5}$, Ronald C. Petersen, MD, PhD ${ }^{6}$, Brit Mollenhauer, \\ MD $^{7}$, Charles H. Adler, MD, PhD $^{8}$, Karen Marder, MD $^{9}$, Caroline H. Williams-Gray, MRCP, \\ $\mathbf{P h D}^{10}$, Dag Aarsland, MD, PhD ${ }^{11}$, Jaime Kulisevsky, MD, PhD $^{12}$, Maria C. Rodriguez-Oroz, \\ MD, PhD ${ }^{13}$, David J. Burn, MD, FRCP ${ }^{14}$, Roger A. Barker, BSc, MBBS, MRCP, PhD $^{10}$, and \\ Murat Emre, MD ${ }^{15}$
}

${ }^{1}$ Division of Movement Disorders, Department of Neurology, University of Louisville, Louisville, Kentucky, USA; and Movement Disorders Program, Frazier Rehab Neuroscience Institute, Louisville, Kentucky, USA ${ }^{2}$ Department of Neurological Sciences, Section of Parkinson Disease and Movement Disorders, Rush University Medical Center, Chicago, Illinois, USA ${ }^{3}$ Department of Neurology, University of North Carolina at Chapel Hill School of Medicine, Chapel Hill, North Carolina, USA 4 Department of Psychology, University of Amsterdam, Amsterdam, The Netherlands; and Department of Neurology, Academic Medical Center, Amsterdam, The Netherlands ${ }^{5}$ Departments of Psychiatry and Neurology, University of Pennsylvania School of Medicine Philadelphia, Pennsylvania, USA; and Parkinson's Disease and Mental Illness Research, Education and Clinical Centers, Philadelphia Veterans Affairs Medical Center, Philadelphia, Pennsylvania, USA 6 Mayo Alzheimer's Disease Research Center, Mayo Clinic, Rochester, Minnesota, USA ${ }^{7}$ Paracelsus-Elena-Klinik, Kassel, Germany; and Georg-August University Goettingen, Goettingen, Germany ${ }^{8}$ Parkinson's Disease and Movement Disorders Center, Department of Neurology, Mayo Clinic, Scottsdale, Arizona, USA ${ }^{9}$ Taub Institute on Alzheimers Disease and the Aging Brain, Department of Neurology, New York Presbyterian Hospital, Columbia University Medical Center, NY, New York, USA ${ }^{10}$ Cambridge Center for Brain Repair, Department of Clinical Neurosciences, University of Cambridge, Cambridge, UK

\footnotetext{
(C) 2012 Movement Disorder Society

"Correspondence to: Irene Litvan, MD, Tasch Endowed Professor in Parkinson Disease, Research Director of the Movement Disorders Program UC San Diego, Department of Neurosciences, University of California San Diego, Movement Disorder Program, 8950 Villa La Jolla Drive Suite C112, La Jolla, CA 92037, USA; ilitvan@ucsd.edu.

Relevant conflicts of interest/financial disclosures: A.I.T. has held consultancies with Medtronic, St. Jude Medical, and Boston Scientific, has served on the advisory board of St. Jude Medical, has received honoraria from Medtronic and the Parkinson's Disease Foundation, and has been awarded grants from Medtronic, GlaxoSmithKline, and the National Parkinson Foundation; D.W. has served on the advisory board of Teva Pharmaceuticals and has received honoraria from The Michael J. Fox Foundation for Parkinson's Research; R.P. has held consultancies with GE Healthcare and Elan Pharmaceuticals, has served on the advisory boards of Pfizer and Janssen Alzheimer Immunotherapy, has been awarded grants from the National Institutes of Health (P50 AG016574 and U01 AG006786), and has received royalties from Oxford University Press; C.H.A. has held consultancies with Merck Serono and Teva Pharmaceuticals; K.M. has been awarded grants from the National Institutes of Health (NS36630 and RR024156); D.B. has received honoraria from Merck Serono and The Michael J. Fox Foundation for Parkinson's Research and has received royalties from Henry Stewart Talks; R.B. has held consultancies with Oxford Biomedica, has served on the advisory board of Teva Lundbeck, has received honoraria from GlaxoSmithKline, has been awarded grants from Parkinson's UK, the European Union FP7 program, the Child Health and Development Institute, and The Michael J. Fox Foundation for Parkinson's Research, has been employed by the University of Cambridge, has received royalties from Wiley and Cambridge University Press, and has received monetary compensation from Springer for editorial work for the Journal of Neurology; and M.E. has held a consultancy with Merck Serono, has served on the advisory board of Merck Serono, has received honoraria from Merck Serono, and has received a clinical study grant from Teva Lundbeck.

Full financial disclosures and author roles may be found in the online version of this article.

Additional Supporting Information may be found in the online version of this article.
} 
${ }^{11}$ Karolinska Institute, Stockholm, Center for Age-Related Medicine, Stavanger University Hospital, Stockholm, Sweden; and Akershus University Hospital/University of Oslo, Oslo, Norway ${ }^{12}$ Movement Disorders Unit, Neurology Department, Sant Pau Hospital and Institute of Biomedical Research (IIB Sant Pau), Universitat Autònoma de Barcelona, Barcelona, Spain; and Centro de Investigación Biomédica en Red sobre Enfermedades Neurodegenerativas, Barcelona, Spain ${ }^{13}$ Department of Neurology, Hospital Donostia, Donostia, Spain; Neuroscience Unit, BioDonostia Research Institute, San Sebatian, San Sebatian, Spain; Ikerbasque, Basque Foundation for Science, Bilbao, Bilbao, Spain; and Centro de Investigación Biomédica en Red sobre Enfermedades Neurodegenerativas, Barcelona, Spain ${ }^{14}$ Institute for Aging and Health, Newcastle University, Newcastle upon Tyne, UK ${ }^{15}$ Behavioral Neurology and Movement Disorders Unit, Department of Neurology, Istanbul Faculty of Medicine, Istanbul University, Istanbul, Turkey

\section{Abstract}

Mild cognitive impairment is common in nondemented Parkinson's disease (PD) patients and may be a harbinger of dementia. In view of its importance, the Movement Disorder Society commissioned a task force to delineate diagnostic criteria for mild cognitive impairment in PD. The proposed diagnostic criteria are based on a literature review and expert consensus. This article provides guidelines to characterize the clinical syndrome and methods for its diagnosis. The criteria will require validation, and possibly refinement, as additional research improves our understanding of the epidemiology, presentation, neurobiology, assessment, and long-term course of this clinical syndrome. These diagnostic criteria will support future research efforts to identify at the earliest stage those PD patients at increased risk of progressive cognitive decline and dementia who may benefit from clinical interventions at a predementia stage.

\section{Keywords}

Parkinson's disease; cognition; mild cognitive impairment; diagnostic criteria; dementia

In Parkinson's disease (PD) there is a spectrum of cognitive dysfunction, ranging from mild cognitive impairment (MCI) to PD dementia (PDD). A Movement Disorder Society (MDS) commissioned task force recently evaluated the literature pertaining to mild cognitive impairment in PD (PD-MCI), and determined that MCI is common in nondemented PD patients and is associated with increasing age, disease duration, and disease severity. ${ }^{1}$ Moreover, PD-MCI predicts the development of dementia, which can occur in up to $80 \%$ of PD patients over the long term. ${ }^{2-4}$

There has been significant heterogeneity, however, in the definition of PD-MCI and the clinical correlates predicting progression to PDD. ${ }^{1,5} \mathrm{~A}$ uniform definition of PD-MCI is important, because it will help identify (1) the clinical characteristics of the earliest stage of PD cognitive impairment, (2), the best predictors of conversion from PD-MCI to PDD, (3) the effects of PD-MCI on quality of life and day-to-day functioning, (4) a well-characterized patient population and a potential outcome measure for clinical trials, and (5) a useful clinical entity that will allow clinicians, patients, caregivers, and researchers to communicate better, thereby improving patient care and research efforts. Specific criteria for diagnosing $\mathrm{MCI}$ in PD will enable clinicians and researchers to identify patients at increased risk of developing PDD and who may benefit from early interventions. PD-MCI criteria may also facilitate research aimed at determining disease pathogenesis in the earlier stages of disease. 
In view of the importance of early recognition of PD-MCI, the MDS Task Force has delineated diagnostic criteria that include (1) characterization of the clinical syndrome and (2) methods for its diagnosis. The proposed criteria are based on a literature review and expert consensus. They will require validation and possibly refinement as additional research improves our understanding of the epidemiology, presentation, neurobiology, assessment, evolution, and treatment of PD-MCI.

\section{Current Methods of Defining MCI}

Different criteria for defining MCI in the general population have been proposed, and several have undergone revision. ${ }^{6} \mathrm{MCI}$ criteria by Petersen et al. ${ }^{7,8}$ require (1) a subjective complaint of cognitive decline by the patient, preferably corroborated by a reliable source,

(2) minimal effect of the decline on day-to-day functioning and the absence of dementia, and (3) evidence of cognitive abnormalities that cannot be simply attributed to age. Such evidence can be based solely on clinician judgment, although formal neuropsychological testing is deemed helpful. Specific neuropsychological tests and cut-off scores are not stated. Quantitative measurements of function and activities of daily living are not required. Categorization into single-domain, multiple-domain, amnestic, and nonamnestic subtypes is based on the results of neuropsychological testing. Proposed MCI criteria recently developed by the National Institute on Aging and the Alzheimer's Association MCI criteria committee $^{6}$ and the DSM-5 Neurocognitive Disorders Work Group ${ }^{9,10}$ would also allow cognitive decline to be detected by health care providers as an alternative to patient or informant report.

\section{Methodology}

The MDS Task Force first reviewed literature on the epidemiology and phenomenology of PD-MCI. ${ }^{1}$ In December 2010, the MDS Task Force participants held a 2-day in-person meeting to critically review current knowledge on MCI in general and in PD specifically, different PD-MCI subtypes, progression of PD-MCI to PDD, the neurobiology of PD-MCI, the role for biomarkers in the diagnosis of cognitive problems in PD, and methods of cognitive assessment. The primary objective of the task force was to develop criteria for PDMCI that would best differentiate these patients from those with normal cognition or dementia. Participants summarized the information presented during group discussions and submitted it to the task force chair (I.L.), who developed the initial manuscript draft. This draft was reviewed and revised in an iterative process by the task force members between January and June 2011, including an in-person meeting in June 2011. This article summarizes the task force's consensus.

\section{PD-MCI Heterogeneity, Subtypes, and Dementia Risk}

In its first review, the MDS Task Force concluded that PD-MCI is common in nondemented patients (mean prevalence, 27\%; range, 19\%-38\%) and is associated with the subsequent development of PDD. ${ }^{1}$ The clinical profile of PD-MCI is heterogeneous, with a range of cognitive domains affected. Overall, non-amnestic, single-domain impairment (i.e., any single nonmemory domain) is the most common subtype of PD-MCI. ${ }^{1}$

Although few studies have evaluated the relationship between specific PD-MCI subtypes and the development of PDD, there is preliminary evidence from prevalent cohorts that nonamnestic single-domain MCI subtype, ${ }^{11}$ executive deficits, ${ }^{12-14}$ impaired verbal fluency, ${ }^{13,15}$ visuospatial deficits, ${ }^{13}$ and memory and language dysfunction ${ }^{14,16}$ all predict PDD. In a population-based, longitudinal study of incident cases, neuropsychological features with a more posterior cortical basis were associated with incident dementia, 
whereas frontostriatally based deficits were not, thereby suggesting two distinct cognitive syndromes with potentially different etiologies and prognoses. ${ }^{17}$

\section{PD-MCI Criteria}

PD-MCI is a syndrome defined by clinical, cognitive, and functional criteria (Table 1). The proposed MDS Task Force criteria are rooted in the MCI criteria previously described, but modified to address issues relatively specific to PD. The criteria were also designed to be consistent with the MDS proposed PDD criteria and thereby allow transitions between categories of normal cognition, MCI, and dementia. ${ }^{18,19}$ Similarly, the proposed PD-MCI criteria utilize a two-level operational schema depending on the comprehensiveness of neuropsychological testing. ${ }^{19}$ Level I and II categories both represent PD-MCI, but differ regarding method of assessment, level of diagnostic certainty, and extent of clinical characterization.

The criteria are also designed to be applicable to a range of populations, including patients of different ethnic ${ }^{19}$ and educational backgrounds, and be reliable and valid for MCI definition in clinical trials and longitudinal studies wherein MCI is an outcome measure or an inclusion/exclusion criterion. The following sections outline proposed criteria for PDMCI, guidelines for level I and II diagnostic categories, and considerations entertained in developing these criteria.

\section{Inclusion Criteria}

First, the diagnosis of $\mathrm{PD}^{20}$ must be clinically established (Table 1). PD-MCI is characterized by an insidious decline in cognitive abilities caused primarily by the underlying disease process. The cognitive decline may be reported by either the patient or informant, or observed by the clinician. This allows for the greatest sensitivity in detecting cognitive decline. Cognitive deficits should be present on testing, and specific recommendations are presented for diagnosing PD-MCI with subtyping (Appendix A; Tables 2 and 3). Finally, cognitive deficits should not interfere significantly with functional independence.

\section{Exclusion Criteria}

Exclusion criteria include parkinsonism other than idiopathic PD, fulfillment of MDS criteria for PDD, other plausible primary explanations for cognitive deterioration, and other PD-associated comorbidities that, in the opinion of the clinician, may have significantly influenced cognitive testing (see Table 1 for specific examples).

\section{Specific Guidelines for PD-MCI Level I and Level II Categories}

Level I (Abbreviated Assessment, Possible PD-MCI)—The level I category allows for the diagnosis of PD-MCI based on an abbreviated cognitive assessment, because comprehensive testing may not always be practical or available. Level I criteria provide less diagnostic certainty than level II. The requirements for level I are impairment on a scale of global cognitive abilities validated for use in PD (Table 2; and see cut-off guidelines for global cognitive tests ${ }^{21-25}$ ) or impairment on a limited battery of neuropsychological tests (e.g., including only one test per cognitive domain or those assessing fewer than five cognitive domains). Identical to the MDS PDD operational criteria, the relevant cognitive domains are attention and working memory as well as executive, language, memory, and visuospatial functions (see Table 3 for examples of tests to assess these domains). When a limited battery of neuropsychological tests is performed, impairment must be present on at least two tests to diagnose PD-MCI by level I criteria. Level I criteria do not allow complete subtyping of PD-MCI. 
Level II (Comprehensive Assessment)—For the diagnosis of PD-MCI by level II criteria and PD-MCI subtyping, the task force recommends formal, comprehensive neuropsychological testing that includes at least two tests for each of the five cognitive domains previously listed. Impairment should be present on at least two tests, either within a single cognitive domain or across different cognitive domains. Impairment on neuropsychological tests may be demonstrated in several ways: performance between 1 to 2 standard deviations (SDs) below age, education, gender, and culturally appropriate norms, significant decline on serial cognitive testing, or significant decline from estimated premorbid levels. In a patient who does not score 1 SD below the normative mean but who reports a change in cognition and has undergone serial neuropsychological testing, a significant (i.e., at least $1 \mathrm{SD}$ or exceeding the reliable change index [RCI]; Appendices B and C) ${ }^{26}$ deterioration in cognition over time is acceptable to diagnose PD-MCI. The use of two tests in each cognitive domain (minimum of 10 tests) for the level II category addresses all cognitive domains equally, can increase sensitivity, and allow full subtyping of PD-MCI.

\section{Subtype Classification for PD-MCI}

Classification of PD-MCI subtypes is important for research purposes and to explore whether impairments in different cognitive domains have a different neurobiological substrate and course. To classify PD-MCI as single or multiple domain, comprehensive neuropsychological testing with at least two tests per cognitive domain must be performed (level II). Presence of two abnormal tests within a single cognitive domain, with the other domains unimpaired, represents a single-domain subtype. If at least one test in two or more cognitive domains is impaired, then PD-MCI should be subtyped as multiple domain. The decision to require an impairment of at least one test in two or more cognitive domains for PD-MCI multiple domain was based on previous studies ${ }^{22,27}$ that show that more stringent requirements (i.e., a total of four abnormal tests, two tests in two domains) would significantly decrease sensitivity to enhance specificity, ${ }^{28}$ which could adversely affect epidemiological studies. The proposed criteria recommend specification of the affected domain(s), rather than using amnestic or nonamnestic terminology, so that potential differences among subtypes may be better studied. Examples of subtype designation would be PD-MCI single domain (i.e., executive) or PD-MCI multiple domain (i.e., memory, visuospatial). Appendix $\mathrm{C}$ gives examples of applications of PD-MCI criteria.

\section{Discussion}

Diagnostic criteria for PD-MCI are justified for several reasons. Recent studies in incident and cross-sectional PD cohorts have revealed the extent and nature of cognitive dysfunction in nondemented PD patients. ${ }^{1}$ Prospective studies to better understand the course, associated features, and consequences of such cognitive deficits, as well as improved ways to investigate therapeutic interventions in such patients, are underway. For research purposes, uniform criteria will allow clearly defined patient populations to be included in such studies. For clinical purposes, physicians and patients can use this designation to validate that cognitive impairment exists, but not to the extent of being labeled dementia.

\section{Terminology}

The term MCI, as applied to the general population, denotes a state of impaired cognitive function without attribution of underlying etiology. ${ }^{8}$ As such, $\mathrm{MCI}$ is a syndrome and, in many patients, a transitional state. Other terms have also been used to define cognitive impairment in nondemented patients. ${ }^{29}$ The task force chose to use the term PD-MCI, given its current use in PD literature. 


\section{Reports of Cognitive Decline}

Empirical evidence to address how to best capture subjective reports of disability is limited. ${ }^{30,31}$ Given these uncertainties, the task force criteria recommend the inclusion of reports of cognitive decline made by either the patient, informant, or clinician observer to increase the likelihood of capturing a change in cognitive function. However, PD patients and their caregivers may both over- and underreport cognitive impairment, ${ }^{32}$ and instruments that can reliably assess decline are needed.

To diagnose PD-MCI, cognitive decline should occur within the context of clinically diagnosed PD. In some cases, however, the onset of mild cognitive impairment relative to the motor symptoms is historically vague, and in other cases, cognitive symptoms occur concurrently. In these scenarios, differentiating PD-MCI from incipient dementia with Lewy bodies (DLB) can be challenging. More research is needed to establish the boundaries between incipient DLB and PD-MCI. Careful clinical and neuropsychiatric assessments and longitudinal evaluations will be needed to establish accurate diagnoses.

\section{Absence of Significant Functional Decline}

Existing MCI criteria do not specify how to document the absence of marked functional decline (e.g., observation, interview, self-report, or rating scales), but emphasize the absence of significant functional impairment resulting from cognitive impairment as the primary feature that differentiates MCI from dementia. A broad survey of key functional areas, such as management of medications ${ }^{19,33}$ or finances, should be considered, because impairments in different cognitive domains of MCI may be associated with reduced performance in different aspects of day-to-day functioning. ${ }^{34}$ There are challenges in separating contributions of cognitive or motor deficits to functional tasks in PD, and this area requires additional study.

\section{PD-Associated Comorbidities}

Mood disorders, apathy, psychosis, ${ }^{35}$ and rapid eye movement sleep behavior disorder $(\mathrm{RBD})^{36}$ are common in PD and may impair cognition or be associated with dementia development. ${ }^{37}$ Moreover, anxiety and cognitive slowing during "off" periods can adversely affect neuropsychological test performance. ${ }^{38,39}$ Thus, patients with motor fluctuations should ideally be assessed in the "on" state, be devoid of anticholinergic medication (or other drugs known to have an effect on cognition), and not have nonmotor features deemed sufficiently severe to invalidate the results of the neuropsychological evaluation. It is the responsibility of the person administering the neuropsychological instruments to judge the extent to which such symptoms might interfere with the testing.

Although the task force maintains consistency with the MDS PDD criteria, in the proposed PD-MCI criteria, the presence of prominent behavioral features, such as psychosis or severe depression, preclude a reliable diagnosis of PD-MCI. Should these symptoms be present, patients should be retested when they have improved or when symptoms have resolved. Apathy may interfere with testing and frequently co-occurs with PDD. The clinician should judge whether specific cognitive deficits exist in addition to apathy, or whether cognitive impairment is only secondary to reduced motivation or effort. Furthermore, although psychosis, RBD, and apathy have been associated with cognitive impairment or development of PDD, there is insufficient evidence to recommend that the presence of these symptoms strongly supports a diagnosis of PD-MCI. 


\section{Neuropsychological Evaluation}

Cognitive Domains and Tests-Although assigning various cognitive tasks to domains is somewhat arbitrary, it is critical for research purposes. The task force followed the general conventions of clinical neuropsychology and cognitive neuroscience ${ }^{40,41}$ and of cognitive research in PD in particular. ${ }^{5,42-45}$ Examples of tests used in PD for global cognitive abilities and cognitive domains are provided (Tables 2 and 3 ).

The task force recommends the use of at least two tests in each cognitive domain for the diagnosis of PD-MCI (level II). Though the optimal number of neuropsychological tests within a given cognitive domain likely depends on the choice of tests and their sensitivity, Schinka et al. demonstrated that the diagnosis of MCI based on two tests per domain is probably more valid than when one test per domain is used. ${ }^{27}$ An excessive or imbalanced number of tests per domain, however, might bias both diagnosis and subtyping of PD-MCI. Additional research on the optimal number and type of tests per cognitive domain and how this affects PD-MCI classification is needed. The recommendation to use two tests in each of the five cognitive domains is particularly applicable to research studies, because this will allow for full subtyping of PD-MCI and permit greater sensitivity in epidemiological studies. It is anticipated that when sufficient research has been completed, the domains and numbers of tests required for a diagnosis of PD-MCI may be modified to make it easier to apply the criteria in clinical practice.

Assessment of Premorbid Functioning-Whether or not a patient is cognitively impaired is judged against the background of his or her premorbid level of functioning, as well as demographic corrections and the psychometric characteristics of the tests used. If premorbid cognitive evaluation is available, test performance may be compared with previous scores, taking into account measurement error, retest effects, and errors resulting from multiple comparisons.

Information on handling measurement errors with RCIs is provided (see Appendix B).

Estimated premorbid levels may be based on demographic characteristics (e.g., education level and occupation) and reading ability and vocabulary using tests such as the National Adult Reading Test (NART) and Wechsler Test of Adult Reading (WTAR). These tests allow reasonably accurate estimates of verbal intelligence and are quite insensitive to cerebral deterioration in the absence of aphasia or marked dysarthria.

Following the DSM-5 draft criteria for a mild neurocognitive disorder, ${ }^{10}$ stronger evidence of cognitive decline comes from having previous test data on patients. Consequently, the task force advocates neuropsychological evaluation early in the course of PD to establish baseline cognitive abilities. ${ }^{46}$ When such baseline data are available, subsequent scores would ideally be compared to the baseline performance and converted to an $\mathrm{RCI}^{47}$ or similar construct that considers test-retest reliability and potential practice effects.

\section{Cutoffs for Neuropsychological Tests and Domains-Studies of MCI have} commonly utilized a range of cut-off scores at 1 to 2 SDs or more below the mean using normative data. One issue in using a rigid cut-off score, such as $-1.5 \mathrm{SD}$, is that highfunctioning people (e.g., those scoring at least $0.5 \mathrm{SD}$ above the mean relative to an appropriate normative sample premorbidly) would have to experience a decline of $>2$ SDs to meet a -1.5-SD cutoff. Assuming a normal distribution of test scores, $30 \%$ of people scoring $0.5 \mathrm{SD}$ or more above the mean premorbidly would not be detected as having experienced a marked decline in cognition, even with a 1.5-SD decline in performance (i.e., false negatives), whereas $7 \%$ scoring 1.5 or more SD below the mean premorbidly would be falsely assumed to have cognitive decline, even without any change in performance over 
time (i.e., false positives). The requirement for impaired performance on at least two tests reduces the risk for false positives, but not false negatives.

Differences in cut-off scores utilized have led to a wide range of prevalence estimates for PD-MCI, ${ }^{32}$ and one study found that the best criterion to minimize the inclusion of cognitively normal patients as having MCI was to require deficits of at least $-1.5 \mathrm{SD}$ in two scores within any single domain (resulting in $30 \%$ PD-MCI) or deficits of at least $-1.5 \mathrm{SD}$ in two scores from different domains (37\% PD-MCI) ${ }^{28}$ The proposed PD-MCI criteria are consistent with these cut-off scores, ${ }^{28}$ but because of potential differences among tests and premorbid patient function, we propose a less-rigid recommendation of a range of 1 to 2 SDs below appropriate norms.

Motor Impairment and Neuropsychological Testing-Some of the recommended neuropsychological tests contain relatively simple conditions that require timed motor responses. Standard scores of these simple conditions may be used to take pure motor slowing into account ${ }^{48}$ (Table 3). Nonetheless, it is recommended that patients be tested in their optimal motor state, using tests that minimize motor demands.

Incorporation of Biomarkers in Criteria-Molecular and imaging biomarkers have been studied in PD-associated cognitive impairment. ${ }^{17,49-56}$ Most studies, however, have been small and have lacked detailed neuropsychological testing, prospective longitudinal evaluations, and independently validated data. Therefore, the task force does not recommend the inclusion of biomarkers as part of the PD-MCI criteria at this time, although future validation of biomarker candidates is highly desired and may lead to revised criteria. An anticipated biomarker for PD-MCI should be detectable at diagnosis, easily and widely accessible, and validated in independent cohorts. There is a need for prospective, longitudinal assessments of accessible biomarkers (including cerebrospinal fluid, blood, and neuroimaging) that also incorporate neuropathological correlation. Though the National Institute on Aging Alzheimer's Association MCI criteria committee has incorporated biomarkers into research criteria for MCI and Alzheimer's disease, the field of PD has not advanced sufficiently to consider biomarkers as criteria for cognitive decline at present.

To summarize, there are several differences between the diagnostic criteria proposed by the MDS and other established criteria, such as Petersen's original MCI criteria: (1) MCI is defined within the context of an existing etiology, namely PD; (2) PD-MCI includes not just "memory" complaints, but also other cognitive changes; (3) cognitive decline can be noted by different sources; (4) PD-MCI must have deficits on either formal neuropsychological testing or a test of global cognitive abilities; (5) specific level I and II categories are outlined, including the number of domains, tests per domain, and cut-off scores suggested; and (6) subtyping is recommended only for evaluations in which two neuropsychological tests for each of the five domains are assessed and is strongly suggested for research purposes.

In conclusion, the proposed MDS Task Force PD-MCI criteria provide a uniform method by which to characterize and diagnose $\mathrm{MCI}$ in $\mathrm{PD}$, providing a framework to advance our understanding of the epidemiology, presentation, neurobiology, assessment, and treatment of PD-MCI. Studies are needed to validate the proposed PD-MCI criteria, and further refinement may be necessary as additional research on PD-MCI is conducted.

\section{References}

1. Litvan I, Aarsland D, Adler CH, et al. MDS task force on mild cognitive impairment in Parkinson's disease: critical review of PD-MCI. Mov Disord. 2011; 26:1814-1824. [PubMed: 21661055] 
2. Hely MA, Reid WG, Adena MA, Halliday GM, Morris JG. The Sydney multicenter study of Parkinson's disease: the inevitability of dementia at 20 years. Mov Disord. 2008; 23:837-844. [PubMed: 18307261]

3. Aarsland D, Tandberg E, Larsen JP, Cummings JL. Frequency of dementia in Parkinson disease. Arch Neurol. 1996; 53:538-542. [PubMed: 8660156]

4. Aarsland D, Andersen K, Larsen JP, Lolk A, Kragh-Sorensen P. Prevalence and characteristics of dementia in Parkinson disease: an 8-year prospective study. Arch Neurol. 2003; 60:387-392. [PubMed: 12633150]

5. Aarsland D, Bronnick K, Williams-Gray C, et al. Mild cognitive impairment in Parkinson disease: a multicenter pooled analysis. Neurology. 2010; 75:1062-1069. [PubMed: 20855849]

6. Albert MS, Dekosky ST, Dickson D, et al. The diagnosis of mild cognitive impairment due to Alzheimer's disease: recommendations from the National Institute on Aging and Alzheimer's Association workgroup. Alzheimers Dement. 2011; 7:1-10. [PubMed: 21255738]

7. Petersen RC. Mild cognitive impairment as a diagnostic entity. J Intern Med. 2004; 256:183-194. [PubMed: 15324362]

8. Petersen RC, Roberts RO, Knopman DS, et al. Mild cognitive impairment: ten years later. Arch Neurol. 2009; 66:1447-1455. [PubMed: 20008648]

9. Jeste, D.; Blacker, D.; Blazer, D., et al. Neurocognitive disorders: a proposal from the DSM-5 Neurocognitive Disorders Work Group. 2010. Available at: www.dsm5.org/Proposed\%20Revision \%20Attachments/APA\%20Neurocognitive\%20Disorders\%20Proposal\%20for\%20DSM-5.pdf

10. Ganguli M, Blacker D, Blazer DG, et al. Classification of neurocognitive disorders in DSM-5: a work in progress. Am J Geriatr Psychiatry. 2011; 19:205-210. [PubMed: 21425518]

11. Janvin CC, Larsen JP, Aarsland D, Hugdahl K. Subtypes of mild cognitive impairment in Parkinson's disease: progression to dementia. Mov Disord. 2006; 21:1343-1349. [PubMed: 16721732]

12. Janvin CC, Aarsland D, Larsen JP. Cognitive predictors of dementia in Parkinson's disease: a community-based, 4-year longitudinal study. J Geriatr Psychiatry Neurol. 2005; 18:149-154. [PubMed: 16100104]

13. Mahieux F, Fenelon G, Flahault A, Manifacier MJ, Michelet D, Boller F. Neuropsychological prediction of dementia in Parkinson's disease. J Neurol Neurosurg Psychiatry. 1998; 64:178-183. [PubMed: 9489527]

14. Levy G, Jacobs DM, Tang MX, et al. Memory and executive function impairment predict dementia in Parkinson's disease. Mov Disord. 2002; 17:1221-1226. [PubMed: 12465060]

15. Jacobs DM, Marder K, Cote LJ, Sano M, Stern Y, Mayeux R. Neuropsychological characteristics of preclinical dementia in Parkinson's disease. Neurology. 1995; 45:1691-1696. [PubMed: 7675228]

16. Hobson P, Meara J. Risk and incidence of dementia in a cohort of older subjects with Parkinson's disease in the United Kingdom. Mov Disord. 2004; 19:1043-1049. [PubMed: 15372593]

17. Williams-Gray CH, Evans JR, Goris A, et al. The distinct cognitive syndromes of Parkinson's disease: 5 year follow-up of the CamPaIGN cohort. Brain. 2009; 132:2958-2969. [PubMed: 19812213]

18. Emre M, Aarsland D, Brown R, et al. Clinical diagnostic criteria for dementia associated with Parkinson's disease. Mov Disord. 2007; 22:1689-1707. [PubMed: 17542011]

19. Dubois B, Burn D, Goetz C, et al. Diagnostic procedures for Parkinson's disease dementia: recommendations from the movement disorder society task force. Mov Disord. 2007; 22:23142324. [PubMed: 18098298]

20. Gibb WR, Lees AJ. The relevance of the Lewy body to the pathogenesis of idiopathic Parkinson's disease. J Neurol Neurosurg Psychiatry. 1988; 51:745-752. [PubMed: 2841426]

21. Hoops S, Nazem S, Siderowf AD, et al. Validity of the MoCA and MMSE in the detection of MCI and dementia in Parkinson disease. Neurology. 2009; 73:1738-1745. [PubMed: 19933974]

22. Dalrymple-Alford JC, MacAskill MR, Nakas CT, et al. The MoCA: well-suited screen for cognitive impairment in Parkinson disease. Neurology. 2010; 75:1717-1725. [PubMed: 21060094]

23. Villeneuve S, Rodrigues-Brazete J, Joncas S, Postuma RB, Latreille V, Gagnon JF. Validity of the Mattis Dementia Rating Scale to detect mild cognitive impairment in Parkinson's disease and 
REM sleep behavior disorder. Dement Geriatr Cogn Disord. 2011; 31:210-217. [PubMed: 21474929]

24. Pagonabarraga J, Kulisevsky J, Llebaria G, Garcia-Sanchez C, Pascual-Sedano B, Gironell A. Parkinson's disease-cognitive rating scale: a new cognitive scale specific for Parkinson's disease. Mov Disord. 2008; 23:998-1005. [PubMed: 18381647]

25. Rossetti HC, Lacritz LH, Cullum CM, Weiner MF. Normative data for the Montreal Cognitive Assessment (MoCA) in a population-based sample. Neurology. 2011; 77:1272-1275. [PubMed: 21917776]

26. Jacobson NS, Truax P. Clinical significance: a statistical approach to defining meaningful change in psychotherapy research. J Consult Clin Psychol. 1991; 59:12-19. [PubMed: 2002127]

27. Schinka JA, Loewenstein DA, Raj A, et al. Defining mild cognitive impairment: impact of varying decision criteria on neuropsychological diagnostic frequencies and correlates. Am J Geriatr Psychiatry. 2010; 18:684-691. [PubMed: 21399729]

28. Dalrymple-Alford JC, Livingston L, Macaskill MR, et al. Characterizing mild cognitive impairment in Parkinson's disease. Mov Disord. 2011; 26:629-636. [PubMed: 21287603]

29. Davis HS, Rockwood K. Conceptualization of mild cognitive impairment: a review. Int J Geriatr Psychiatry. 2004; 19:313-319. [PubMed: 15065223]

30. Brown RG, MacCarthy B, Jahanshahi M, Marsden CD. Accuracy of self-reported disability in patients with parkinsonism. Arch Neurol. 1989; 46:955-959. [PubMed: 2528339]

31. Naismith SL, Pereira M, Shine JM, Lewis SJ. How well do caregivers detect mild cognitive change in Parkinson's disease? Mov Disord. 2010; 26:161-164. [PubMed: 20818607]

32. Caviness JN, Driver-Dunckley E, Connor DJ, et al. Defining mild cognitive impairment in Parkinson's disease. Mov Disord. 2007; 22:1272-1277. [PubMed: 17415797]

33. Dujardin K, Dubois B, Tison F, et al. Parkinson's disease dementia can be easily detected in routine clinical practice. Mov Disord. 2010; 25:2769-2776. [PubMed: 20925065]

34. Bangen KJ, Jak AJ, Schiehser DM, et al. Complex activities of daily living vary by mild cognitive impairment subtype. J Int Neuropsychol Soc. 2010; 16:630-639. [PubMed: 20374675]

35. Aarsland D, Andersen K, Larsen JP, et al. The rate of cognitive decline in Parkinson disease. Arch Neurol. 2004; 61:1906-1911. [PubMed: 15596611]

36. Vendette M, Gagnon JF, Decary A, et al. REM sleep behavior disorder predicts cognitive impairment in Parkinson disease without dementia. Neurology. 2007; 69:1843-1849. [PubMed: 17984452]

37. Mavandadi S, Nazem S, Ten Have TR, et al. Use of latent variable modeling to delineate psychiatric and cognitive profiles in Parkinson disease. Am J Geriatr Psychiatry. 2009; 17:986995. [PubMed: 19855199]

38. Peralta C, Stampfer-Kountchev M, Karner E, et al. Orthostatic hypotension and attention in Parkinson's disease with and without dementia. J Neural Transm. 2007; 114:585-588. [PubMed: 17195917]

39. Witjas T, Kaphan E, Azulay JP, et al. Nonmotor fluctuations in Parkinson's disease: frequent and disabling. Neurology. 2002; 59:408-413. [PubMed: 12177375]

40. Lezak, MD.; Howieson, DB.; Loring, DW. Neuropsychological Assessment. 4. New York: Oxford University Press: Oxford University Press; 2004.

41. Gazzaniga, MS.; Ivry, RB.; Mangun, GR. Cognitive Neuroscience: The Biology of the Mind. 3. New York: W.W. Norton; 2009.

42. Caballol N, Marti MJ, Tolosa E. Cognitive dysfunction and dementia in Parkinson disease. Mov Disord. 2007; 22(Suppl 17):S358-S366. [PubMed: 18175397]

43. Muslimovic D, Schmand B, Speelman JD, de Haan RJ. Course of cognitive decline in Parkinson's disease: a meta-analysis. J Int Neuropsychol Soc. 2007; 13:920-932. [PubMed: 17942010]

44. Tröster AI. Neuropsychological characteristics of dementia with Lewy bodies and Parkinson's disease with dementia: differentiation, early detection, and implications for "mild cognitive impairment” and biomarkers. Neuropsychol Rev. 2008; 18:103-119. [PubMed: 18322801] 
45. Bothe MR, Uttner I, Otto M. Sharpening the boundaries of Parkinson-associated dementia: recommendation for a neuropsychological diagnostic procedure. J Neural Transm. 2010; 117:353367. [PubMed: 20119649]

46. Foltynie T, Brayne CE, Robbins TW, Barker RA. The cognitive ability of an incident cohort of Parkinson's patients in the UK. The CamPaIGN study Brain. 2004; 127:550-560.

47. Tröster AI, Woods SP, Morgan EE. Assessing cognitive change in Parkinson's disease: development of practice effect-corrected reliable change indices. Arch Clin Neuropsychol. 2007; 22:711-718. [PubMed: 17644304]

48. Arbuthnott K, Frank J. Trail making test, part B as a measure of executive control: validation using a set-switching paradigm. J Clinical Exp Neuropsychology. 2000; 22:518-528.

49. Mollenhauer B, Trenkwalder C. Neurochemical biomarkers in the differential diagnosis of movement disorders. Mov Disord. 2009; 24:1411-1426. [PubMed: 19412961]

50. Mollenhauer B, Trenkwalder C, von Ahsen N, et al. Beta-amlyoid 1-42 and tau-protein in cerebrospinal fluid of patients with Parkinson's disease dementia. Dement Geriatr Cogn Disord. 2006; 22:200-208. [PubMed: 16899997]

51. Siderowf A, Xie SX, Hurtig H, et al. CSF amyloid \{beta> 1-42 predicts cognitive decline in Parkinson disease. Neurology. 2010; 75:1055-1061. [PubMed: 20720189]

52. Foltynie T, Lewis SG, Goldberg TE, et al. The BDNF Val66Met polymorphism has a gender specific influence on planning ability in Parkinson's disease. J Neurol. 2005; 252:833-838. [PubMed: 15772739]

53. Guerini FR, Beghi E, Riboldazzi G, et al. BDNF Val66Met polymorphism is associated with cognitive impairment in Italian patients with Parkinson's disease. Eur J Neurol. 2009; 16:12401245. [PubMed: 19538209]

54. Gattellaro G, Minati L, Grisoli M, et al. White matter involvement in idiopathic Parkinson disease: a diffusion tensor imaging study. Am J Neuroradiol. 2009; 30:1222-1226. [PubMed: 19342541]

55. Alves G, Bronnick K, Aarsland D, et al. CSF amyloid-beta and tau proteins, and cognitive performance, in early and untreated Parkinson's disease: the Norwegian ParkWest study. J Neurol Neurosurg Psychiatry. 2010; 81:1080-1086. [PubMed: 20547614]

56. Setó-Salvia N, Clarimon J, Pagonabarraga J, et al. Dementia risk in Parkinson disease: disentangling the role of MAPT haplotypes. Arch Neurol. 2011; 68:359-364. [PubMed: 21403021]

57. National Institute of Neurological Disorders and Stroke. NINDS common data elements. 2011. Available at: www.ninds.nih.gov/research/clinical_research/toolkit/common_data_elements.htm

58. Gill DJ, Freshman A, Blender JA, Ravina B. The Montreal cognitive assessment as a screening tool for cognitive impairment in Parkinson's disease. Mov Disord. 2008; 23:1043-1046. [PubMed: 18381646]

59. Kulisevsky J, Pagonabarraga J. Cognitive impairment in Parkinson's disease: tools for diagnosis and assessment. Mov Disord. 2009; 24:1103-1110. [PubMed: 19353727]

60. Verbaan D, Marinus J, Visser M, et al. Cognitive impairment in Parkinson's disease. J Neurol Neurosurg Psychiatry. 2007; 78:1182-1187. [PubMed: 17442759]

61. Llebaria G, Pagonabarraga J, Kulisevsky J, et al. Cut-off score of the Mattis Dementia Rating Scale for screening dementia in Parkinson's disease. Mov Disord. 2008; 23:1546-1550. [PubMed: 18546326] 


\section{TABLE 1}

\section{Criteria for the Diagnosis of PD-MCI}

I. Inclusion criteria

- Diagnosis of Parkinson's disease as based on the UK PD Brain Bank Criteria ${ }^{20}$

- Gradual decline, in the context of established PD, in cognitive ability reported by either the patient or informant, or observed by the clinician

- Cognitive deficits on either formal neuropsychological testing or a scale of global cognitive abilities (detailed in section III)

- Cognitive deficits are not sufficient to interfere significantly with functional independence, although subtle difficulties on complex functional tasks may be present

\section{Exclusion criteria}

- Diagnosis of PD dementia based on MDS Task Force proposed criteria ${ }^{18}$

- Other primary explanations for cognitive impairment (e.g., delirium, stroke, major depression, metabolic abnormalities, adverse effects of medication, or head trauma)

- Other PD-associated comorbid conditions (e.g., motor impairment or severe anxiety, depression, excessive daytime sleepiness, or psychosis) that, in the opinion of the clinician, significantly influence cognitive testing

III. Specific guidelines for PD-MCI level I and level II categories

A. Level I (abbreviated assessment)

- Impairment on a scale of global cognitive abilities validated for use in $\mathrm{PD}^{a}$ or

- Impairment on at least two tests, when a limited battery of neuropsychological tests is performed (i.e., the battery includes less than two tests within each of the five cognitive domains, or less than five cognitive domains are assessed)

B. Level II (comprehensive assessment)

- Neuropsychological testing that includes two tests within each of the five cognitive domains (i.e., attention and working memory, executive, language, memory, and visuospatial $)^{b}$

- Impairment on at least two neuropsychological tests, represented by either two impaired tests in one cognitive domain or one impaired test in two different cognitive domains

- Impairment on neuropsychological tests may be demonstrated by:

$$
\begin{array}{ll}
\text { - } & \text { Performance approximately } 1 \text { to } 2 \text { SDs below appropriate norms or } \\
\text { - } & \text { Significant decline demonstrated on serial cognitive testing or } \\
\text { - } & \text { Significant decline from estimated premorbid levels }
\end{array}
$$

\section{Subtype classification for PD-MCI (optional, requires two tests for each of the five cognitive domains assessed and is} strongly suggested for research purposes) ${ }^{c}$

- $\quad$ PD-MCI single-domain - abnormalities on two tests within a single cognitive domain (specify the domain), with other domains unimpaired or

- $\quad$ PD-MCI multiple-domain —abnormalities on at least one test in two or more cognitive domains (specify the domains)

\footnotetext{
${ }^{a}$ See Table 2. Examples of scales of global cognitive abilities validated in PD.

${ }^{b}$ See Table 3. Examples of neuropsychological tests for the five cognitive domains.

${ }^{c}$ Subtype classifications are applicable only to those PD-MCI who have had at least two tests within each of the five cognitive domains administered.
} 
TABLE 2

Examples of Neuropsychological Scales for Assessing Global Cognitive Abilities and Estimating Premorbid Intelligence $^{*}$

\begin{tabular}{|c|c|c|}
\hline Assessment & Neuropsychological Tests ${ }^{40}$ & Estimated Time of Test, min \\
\hline \multirow[t]{4}{*}{ Global cognition } & $\mathrm{MoCA}^{22,58}$ & 10 \\
\hline & PD-CRS ${ }^{24,59}$ & 15 \\
\hline & SCOPA-COG 59,60 & 15 \\
\hline & $\operatorname{MDRS}^{23,59,61}$ & 20 to 30 \\
\hline \multirow[t]{2}{*}{ Estimated premorbid intelligence } & NART $^{40}$ & 5 \\
\hline & WTAR $^{40}$ & 5 \\
\hline
\end{tabular}

Some of these tests are copyrighted and require permission from the authors to be used. The PD-CRS is a scale owned by the Movement Disorders Society (see www.movementdisorders.org/publications/rating_scales/).

See also reference no. 57.

Abbreviations: MoCA, Montreal Cognitive Assessment; PD-CRS, Parkinson's Disease-Cognitive Rating scale; SCOPA-COG, Scales for Outcomes of Parkinson's disease-Cognition; MDRS, Mattis Dementia Rating Scale. 
TABLE 3

Examples of Tests for Cognitive Domains and Estimated Time*

\begin{tabular}{|c|c|c|}
\hline Cognitive Domain & Neuropsychological Tests ${ }^{40}$ & $\begin{array}{l}\text { Estimated } \\
\text { Time of Test, } \\
\text { min }\end{array}$ \\
\hline \multirow[t]{5}{*}{ Attention and working memory } & WAIS-IV (or earlier version) Letter Number Sequencing & 5 \\
\hline & WAIS-IV Coding (or earlier version) or other substitution task, written or oral & 5 \\
\hline & Trail Making Test ${ }^{a}$ & 5 to 10 \\
\hline & Digit span backward or digit ordering & 5 \\
\hline & Stroop color-word test & 5 to 10 \\
\hline \multirow[t]{3}{*}{ Executive function } & Wisconsin Card Sorting Test (CST), or modified CST (Nelson's modification) & 15 \\
\hline & Tower of London test-Drexel version, or Stockings of Cambridge (CANTAB) & 10 to 15 \\
\hline & $\begin{array}{l}\text { Verbal fluency test, such as letter fluency (COWAT or similar tests), category fluency } \\
\text { (animals, supermarket, or similar), or alternating fluency tasks (if a well-standardized } \\
\text { version is used). Not more than one verbal fluency test abnormality should be used to } \\
\text { satisfy the MCI criterion of two abnormal test performances because of the strong } \\
\text { relationship among these tests; } 10 \text { points Clock Drawing Test }\end{array}$ & 5 \\
\hline \multirow[t]{2}{*}{ Language } & WAIS-IV (or earlier version) Similarities & 10 to 15 \\
\hline & $\begin{array}{l}\text { Confrontation naming task, such as Boston Naming Test (or short-form validated in PD) } \\
\text { or Graded Naming Test }\end{array}$ & 5 to 15 \\
\hline \multirow[t]{3}{*}{ Memory $b$} & $\begin{array}{l}\text { Word list learning test with delayed recall and recognition conditions, such as Rey's } \\
\text { Auditory Verbal Learning Test, California Verbal Learning Test, Hopkins Verbal } \\
\text { Learning Test, and Selective Reminding Test }\end{array}$ & 10 to 20 \\
\hline & $\begin{array}{l}\text { Prose recall }{ }^{3} \text { test with a delayed recall condition, such as Wechsler Memory Scale-IV } \\
\text { Logical Memory subtest (or earlier version) or Rivermead Behavioural Memory Test } \\
\text { paragraph recall subtest }\end{array}$ & 10 to 15 \\
\hline & Brief Visuospatial Memory Test-Revised (BVMT-R) & 10 to 15 \\
\hline \multirow[t]{3}{*}{ Visuospatial $^{c}$ function } & Benton's Judgment of Line Orientation & 5 to 10 \\
\hline & Hooper Visual Organization Test & 10 \\
\hline & Clock copying (e.g., Royall's CLOX) & 5 \\
\hline
\end{tabular}

Two highly similar tests (e.g., two list learning tests or two story recall tests) should not be used to meet the MCI criterion of two test-score abnormalities. Similarly, highly correlated scores from the same test (e.g., immediate and delayed recall of a word list) should not be used to meet the criterion of two test abnormalities. Abnormality of two highly correlated scores only increases confidence in the existence of impairment.

*

See also reference no. 40 for references.

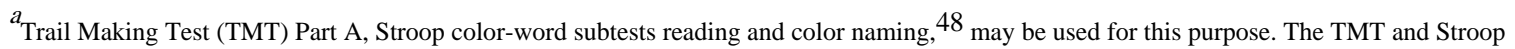
subtests are timed and give an estimate of manual and articulatory slowness that can be used to judge the significance of the more demanding conditions (e.g., TMT-B, Stroop interference) or of other tests, such as fluency performance. Oral versions of neuropsychological tests may provide alternatives.

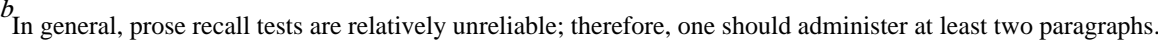

${ }^{c}$ Most visual memory tests are less appropriate for PD-MCI because these tests either require motor responses (e.g., drawing of geometric or other figures) or evaluate recognition memory, which is less sensitive to early memory decline. BVMT-R is an exception because it allows for assessment of the motor component via the copy condition. 